\title{
Daniel F. Bridges 2001: \\ Daniel Mathematical Connections in Art, Music and Science \\ June 2001, Winfield, Kansas, USA.
}

The fourth annual Bridges Conference took place at Southwestern College in Winfield, Kansas, USA, in June 2001. Highlights of the year's conference included the presentation to the college of a sculpture titled "Genesis" by sculptor and conferee Brent Collins. The piece stands in front of the Beech Science Center on the campus. The college owns one other sculpture titled "Pax Mundi" by Collins. This work is located in the foyer of the mathematics department.

Collins, an independent artist, and Carlo Sequin, from the computer department of the University of California at Berkeley, have collaborated on the mathematical implications of Collins's intuitive sculptures. Sequin investigates the mathematics of the sculptures and produces three-dimensional models through a process known as sterolithography. Sequin exhibited some of these models and spoke on the second day of the conference.

Ivars Peterson, mathematics columnist for Science News and author of The Mathematical Tourist and Islands of Truth, among several other books, presented from a manuscript he is preparing on mathematics and art. He discussed the mathematical implications of public art in Toronto, Canada; Washington DC, USA, and New Orleans, Louisiana, USA. In addition, Peterson has commented on many of the artists and mathematicians who spoke at the conference in his column.

Leonard Shlain, a laparoscopic surgeon with the California Pacific Medical Center in San Francisco, California, presented on his thesis that public symbol systems challenged a feminine orientation in prehistoric cultures. This idea is developed fully in his book The Alphabet versus the Goddess. He is best known by conferees for his book Art and Physics, demonstrating parallel and causative developments in the two fields.

For the fourth year, the conference enjoyed the performances and commentary by Corey Cerovsek. A world class violinist, Cerovsek worked on doctoral degrees in music and in mathematics at Indiana University. He had completed coursework for both degrees by the age of eighteen. He performed after each of the three morning sessions and during several of the after dinner gatherings. On the final evening, Corey joined with mathematicians and musicians to play at the conference finale. He often played and then demonstrated and commented on the mathematical structure of the music he performed. Works by Bach and Barber were among the most popular selections.

In addition, Don Crowe from the University of Wisconsin-Madison presented on "Symmetries of Culture," the title of his classic text. Craig Kaplan from the University 
of Washington, Seattle talked about the construction of "Symmetrohedra." Javier Barallo from the University of Basque Country in San Sebastian, Spain talked about "Fractals and Multi-Layered Colorings." Finally one of the several parents of mathematics and arts conferences, Nat Friedman from the State University of New York-Albany, spoke on "Multiple Mobius Band Minimal Surfaces." Friedman was also a major contributor to the public school workshops which followed the formal conference.

A dazzling array of smaller group presentations occurred in the afternoons. From the structure of Native American architectures to tessellations in physical chemistry and from snake robotic research to "functional image synthesis," the conference was lively and varied.

A new feature at the Bridges Conference was an exhibit of mathematical visual art. The Mathematical Visual Art exhibit was held close to the main auditorium to allow attendees to visit the exhibit during breaks. The exhibit coordinator was Dr. Robert Fathauer, the founder of Tessellations Company in Tempe, Arizona.

This year, the conference presented a large number of geometers who brought numerous Polyhedra made from various materials in different sizes as small as a sugar cube and as big as several feet in diameter. They were located in a large hall, which was connected to the art exhibit. During the breaks, the audience had the chance to communicate with the sculptors and to observe the process of constructing some of these geometric objects.

On Saturday evening, the conferees traveled to the CyberDome Theater at Exploration Place in Wichita, Kansas. There they were presented the public program, "Cracking the Cosmic Code," with a discussion of new computer technologies by the staff of the center. Finally a specially designed display of the theater's capabilities was presented with a question and answer session and a brief tour of the facility. The theater itself has technology matched only by the Adler Planetarium in Chicago, Illinois.

Reza Sarhangi, conference director, noted that this was the most successful conference to date. Several conferees deemed it one of the top three in the world. Sarhangi said, "By exploring the bridges between mathematics and the arts, we hope to make a difference in how people feel toward math." Next year's conference will be held at Towson University in Baltimore, Maryland and the year after that - 2003 - the conference will be held in Europe. Future plans call for an effort to expand both the domestic and international number of conferees. In addition, a journal is being considered which will offer interdisciplinary subjects based on the "Bridges" concept. The future looks very bright for an exciting academic approach whose time has arrived. Reza Sarhangi is to be congratulated for his efforts in advancing the work of several fields.

\section{About the reporter}

Daniel F. Daniel received his BA in English Literature from Berea College in Kentucky, his MA in literature from the University of Chicago, and his $\mathrm{PhD}$ in literature and philosophy from the University of Wisconsin-Madison. He has done post doctoral work in Semiotics at Kansas University and in Prosaics at Northwestern University in Evanston, Illinois. He currently serves on the Board of Environmental Ethics at Dade County Community College in Miami, Florida. He is a professor of English Literature and a member of the Integrative Studies faculty at Southwestern College in Winfield, Kansas. 